\title{
Cubierta vegetal con Teramnus labialis en plantaciones citrícolas: efectos sobre algunas propiedades físicas del suelo
}

\section{Cover crop with Teramnus labialis in a citrus orchard: effects on some physical properties of the soil}

\author{
Dayami Fontes Marrero ${ }^{1 *}$; Lázaro E. Pulido Delgado ${ }^{2}$; Nieves Cubillas Iañez ${ }^{3}$; \\ Carlos Mazorra Calero ${ }^{4}$; Manrique Lazo Rodríguez; \\ Lucas Rodríguez Pérez ${ }^{6}$; Leydis Castellano Rodríguez ${ }^{7}$
}

\begin{abstract}
Resumen
El uso de coberturas vivas de leguminosas en plantaciones citrícolas constituye una alternativa viable para el mejoramiento de las propiedades de los suelos, siempre que ellas se manejan adecuadamente en estas áreas. En la universidad de Ciego de Ávila, Cuba se viene trabajando en la evaluación del efecto de coberturas vivas de leguminosas en plantaciones citrícolas en producción, con el objetivo de evaluar el efecto que ejerce la cobertura de leguminosa sobre algunas propiedades del suelo. El trabajo se realizó durante cuatro años (2001-2005) en una plantación de naranja Valencia Late ( Citrus sinensis L. Osbeck) en producción de 22 años, con marco de plantación de 8 X 4 m, plantada sobre un suelo Ferralítico Rojo típico, perteneciente a la CPA "José Martí", en Ciego de Ávila. Se empleó un diseño de bloque al azar, con tres tratamientos: con cobertura de Teramnus labialis $\left(\mathrm{T}_{1}\right)$ con una dosis de siembra 2.5 kg.ha ${ }^{-1}$ a voleo, cobertura natural $\left(\mathrm{T}_{2}\right)$ y suelo desnudo $\left(\mathrm{T}_{3}\right)$. Se determinaron las propiedades estructurofuncionales del suelo, la composición de macroagregados expresadas en el coeficiente de estructura y el porcentaje de agregados estables en agua, densidad del suelo, humedad y la porosidad. Los incrementos en los contenidos de humedad, el volumen específico total de poros y de aire, el coeficiente de estructura, así como el porcentaje de agregados estables al agua, en el suelo, donde se establece la cobertura de Teramnus labialis, demuestran la eficiencia de la cobertura vegetal en estas plantaciones citrícolas.

Palabras claves: Teramnus labialis, porosidad de aireación, volumen total de poros.
\end{abstract}

\footnotetext{
1 Profesora de la Facultad de Ciencias Agropecuaria, Universidad de Ciego de Ávila "Máximo Gómez Báez", Departamento de Producción Agropecuaria, Profesora de Sistema de Producción Animal, Master en Pastos y Forrajes, Doctora en Ciencias Agrícolas.

2 Profesor de la Facultad de Ciencias Agropecuaria, Universidad de Ciego de Ávila "Máximo Gómez Báez", Departamento de Producción Agropecuaria, Profesor de Horticultura, Doctor en Ciencias Agrícolas. dayamif@agronomia.unica.cu

3 Profesora de la Facultad de Ciencias Agropecuaria, Universidad de Ciego de Ávila "Máximo Gómez Báez", Departamento de Producción Agropecuaria, Profesora de Zootecnia, Doctora en Ciencias Agropecuarias.

4 Profesora de la Facultad de Ciencias Agropecuaria, Universidad de Ciego de Ávila "Máximo Gómez Báez", Departamento de Producción Agropecuaria, Profesora de Zootecnia, Doctora en Ciencias Agropecuarias.

5 Profesor de la Facultad de Ciencias Agropecuaria, Universidad de Ciego de Ávila "Máximo Gómez Báez", Departamento de Producción Agropecuaria, Profesor de Fitotecnia general, Ingeniero Agrónomo.

6 Profesor de la Facultad de Ciencias Agropecuaria, Universidad de Ciego de Ávila "Máximo Gómez Báez", Departamento de Producción Agropecuaria, Profesor de Ecología, Master en Agroecología.

7 Profesor de la Facultad de Ciencias Agropecuaria, Universidad de Ciego de Ávila "Máximo Gómez Báez", Departamento de Producción Agropecuaria, Profesor de Ecología, Master en Agroecología

* Autor para correspondencia
} 


\begin{abstract}
The use of leguminous cover crops in citrus orchards constitutes a viable alternative for the improvement of soil properties, whenever they are appropriately managed. In Ciego de Avila University, Cuba, it was evaluated the effect of a leguminous cover crop on some properties of an orchard soil. The work was carried out during four years in an orange plantation of Valencia late (Citrus sinensis L. Osbeck) in a 22 years-old orchard, with a plantation frame of $8 \times 4 \mathrm{~m}$, planted on a typical red Ferralitic soil, belonging to the CPA "José Martí", in Ciego de Avila. It was used a random block design with three treatments: one with covering of Teramnus labialis (T1), one with expontaneous vegetation (T2) and the third with no vegetation (T3). The functional structure properties of the soil were determined, and also the composition of macroaggregates expressed in the structure coefficient and the percentage of stable added in water, soil density, humidity and porosity. The increments in the humidity of the soil, the specific volume of pores and air, the structure coefficient, as well as the percentage of stable added in water, in the soil where the covering of Teramnus labialis was stablished, show the efficiency of cover crops in these citrus orchards.
\end{abstract}

Key words: Teramnus labialis, aireation porosity, total volume of pores.

\section{Introducción}

La utilización comercial de leguminosas forrajeras cuenta con una experiencia acumulada de cientos de años en las condiciones tropicales. En cambio, su empleo como cobertura permanente en frutales no tiene antecedentes a escala comercial en nuestro país. Sólo han sido evaluadas las bondades de la cobertura con leguminosas en las condiciones de suelo Ferralítico rojo típico y con el empleo de agrotóxicos para su establecimiento, por Pérez Carmenate (1998), Borroto et al. (2000), Clavel (2004) y Navia (2005).

Se conoce la capacidad de esta familia vegetal como mejoradora de las condiciones del suelo, a través del reciclaje de nutrientes y la fijación simbiótica del nitrógeno atmosférico. Igualmente es conocida la facultad de muchas especies leguminosas volubles para controlar la vegetación indeseable acompañante en los agroecosistemas tropicales (GUTIÉRREZ et al., 2006). La producción de frutas (cítricas) se basa en sistemas de altos insumos, entre los que se destacan los sistemas de riego, fertilizantes minerales, plaguicidas químicos y altas dosis de herbicidas, al extremo de mantener el suelo desnudo. La utilización de todos esos agrotóxicos provoca altos riesgos de contaminación ambiental.

En tal caso, se hace impostergable la búsqueda de alternativas de producción de alimentos, con tecnologías más armoniosas con el medio ambiente y más económicamente sostenibles.

Una de estas prácticas paliativas lo constituye el uso de coberturas vegetales, las que actualmente están siendo incluidas en algunos sistemas agrícolas con el objetivo de incrementar la fertilidad del suelo y el funcionamiento del cultivo a largo plazo, a partir del control de erosión, incremento de la matería orgánica y mejoramiento de las propiedades físicas del suelo variando su acción en dependencia de las condiciones edafoclimáticas y de cultivos en que se empleen. En el presente trabajo tiene el objetivo de evaluar el efecto que ejerce la cobertura de leguminosa sobre algunas propiedades físicas del suelo.

\section{Materiales y métodos}

El experimento se desarrolló durante los años 2001-2005, en áreas de la Cooperativa de Producción Agropecuaria “José Martí “, en Ceballos, Ciego de Ávila, Cuba, en una plantación de naranja Valencia late (Citrus sinensis L. Osbeck), en producción, con 22 años de edad, establecida a 8 x $4 \mathrm{~m}$ de distancia, sobre un suelo Ferralítico Rojo compactado eútrico (HERNÁNDEZ et al., 1999), correlacionado con Rhodic eutrustox de la Soil Taxonomy (SOIL SURVEY STAFF, 2003) y con Rhodic Ferrasol de 
la World Referente Base (DRIESSEN; DECKERS; NACHTERGAELE, 2001), representando este subtipo de suelo el $67.22 \%$ de los presentes en la Empresa, con $\mathrm{pH}$ medio de 5.7, materia orgánica de $2.80 \%$ y contenido de $\mathrm{P}_{2} \mathrm{O}_{5}$ y $\mathrm{K}_{2} \mathrm{O}$ de 0.80 y 20.5 $\mathrm{mg}$ en $100 \mathrm{~g}$ de suelo, respectivamente. El suelo, de acuerdo a su composición granulométrica se considera arcilloso.

Los datos climáticos que se ofrecen en la Figura 1, provienen de la Estación Agrometeorológica 78346 de Ciego de Ávila, la cual esta ubicada a $\operatorname{los} 21^{\circ} 47^{\prime}$ de Latitud Norte y $78^{\circ} 47^{\prime}$ de Longitud Oeste, a una altura de $26.39 \mathrm{msnm}$.

El experimento fue implantado con tres tratamientos: cobertura de Teramnus labialis $\left(\mathrm{T}_{1}\right)$ con una dosis de siembra $2.5 \mathrm{~kg} \cdot \mathrm{ha}^{-1}$ a voleo, cobertura natural $\left(\mathrm{T}_{2}\right)$ manejada con la vegetación espontánea que se desarrolla dentro de estas plantaciones, donde predomina en más del $60 \%$ Paspalum notatum y suelo desnudo $\left(\mathrm{T}_{3}\right)$.

Se empleó un diseño experimental de bloques al azar con tres repeticiones. Independientemente del tratamiento evaluado, cadaréplica estaba conformada por una parcela de $64 \mathrm{~m}^{2}$, que incluía dos árboles de cítrico y sus dos calles colindantes. La muestra tomada en cada réplica/tratamiento correspondió al $5 \%$ de la parcela, según recomendaciones de PérezCarmenate (1998).

Para lograr el establecimiento de Teramnus labialis, se empleó el sistema de preparación de suelo propuesto por Pérez-Carmenate (1998), consistente en realizar 15 días antes de la siembra una aplicación de herbicida (Glyphosate a dosis de

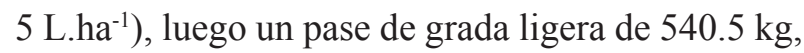
la siembra a voleo y posteriormente una grada ligera para garantizar la incorporación de las semillas.

La siembra de la leguminosa se efectuó en mayo de 2001, escarificando las semillas y sin proceder a la inoculación con Rhizobium, con una densidad de $2.5 \mathrm{~kg} \cdot \mathrm{ha}^{-1}$ de semilla pura germinable (SPG), aplicándose $50 \mathrm{~kg} \cdot$ ha $^{-1}$ de $\mathrm{P}_{2} \mathrm{O}_{5}$ (superfosfato simple como portador mineral), de acuerdo a lo establecido por Pérez y Rolo (1998).

Durante la fase de establecimiento se realizaron tres labores de escardes manuales para el control de las plantas arvenses, al mismo tiempo en que se cortaron las ramas apicales de la leguminosa, evitando que treparan el árbol, hasta lograr un área cubierta por ésta superior al $70 \%$.

\section{Sobre algunas propiedades físicas del suelo}

Las determinaciones de las propiedades físicas evaluadas se realizaron en cada tratamiento, en las que se tomaron muestras de suelo a la profundidad (0.00 - $0.20 \mathrm{~m})$, según establece Lorenzo (1992), transcurrido el primer año experimental y al cuarto año de establecido el experimento. Las variables evaluadas fueron:

- Densidad del suelo $\left(\mathrm{d}_{\mathrm{v}}\right)$ : Se utilizó el método de los cilindros cortantes según Kaúrichev (1984), los cuales se introdujeron en el suelo a las profundidades de estudio, y se determinó posteriormente su contenido de humedad: $d_{v}=\frac{m_{s}}{v_{t}}$

Donde: $\mathrm{m}_{\mathrm{s}}$ : masa de suelo seco; $\mathrm{v}_{\mathrm{t}}$ : volumen total del cilindro

- Densidad de la fase sólida $\left(\rho_{s}\right)$ : Por el método del picnómetro (KAÚRICHEV, 1984), utilizándose como fórmula: $\rho_{s}=\frac{m_{s}}{v_{s}}$

donde: $\mathrm{m}_{\mathrm{s}}=$ masa de suelo seco; $v_{s}=$ volumen de la fase sólida del suelo.

- Humedad a capacidad de campo según Kaúrichev (1984).

Porosidad total o volumen total de poros $(\mathrm{Pt})$ : según Kaúrichev (1984), a través de la ecuación:

$$
P_{t}=\left(1-\frac{\rho_{s}}{d_{v}}\right) \times 100
$$

donde: $\mathrm{d}_{\mathrm{v}}$ : densidad de suelo; $\rho_{s}$ densidad de la fase sólida. 

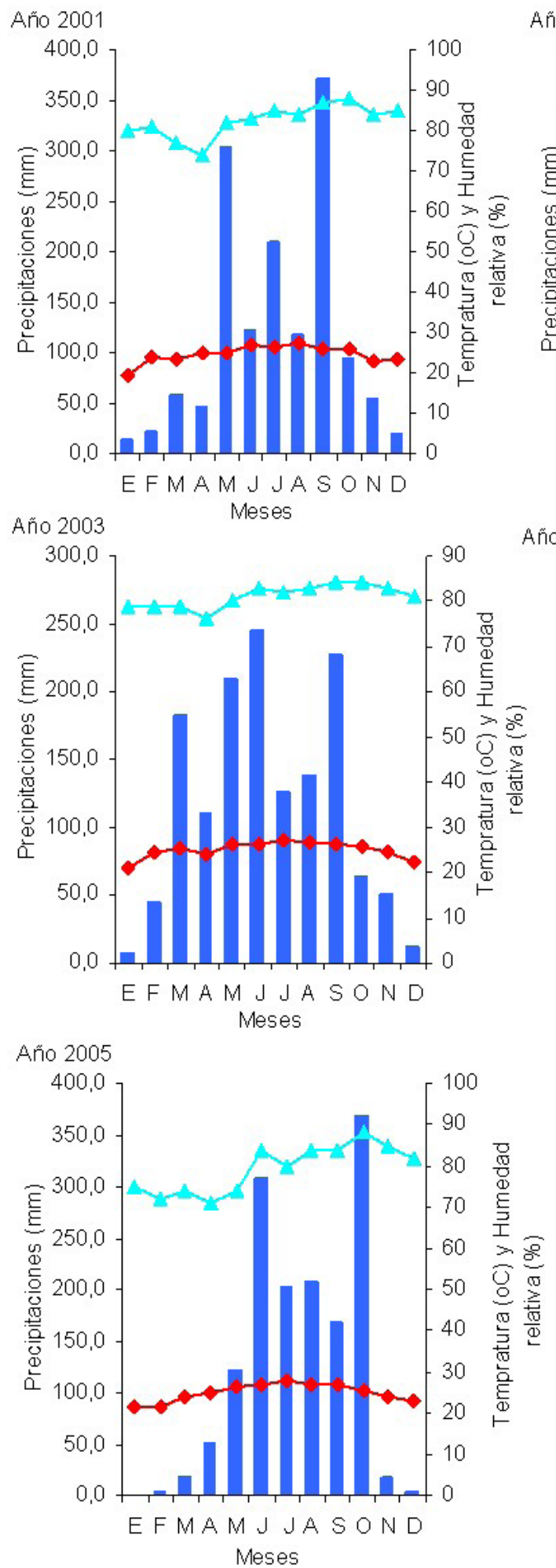

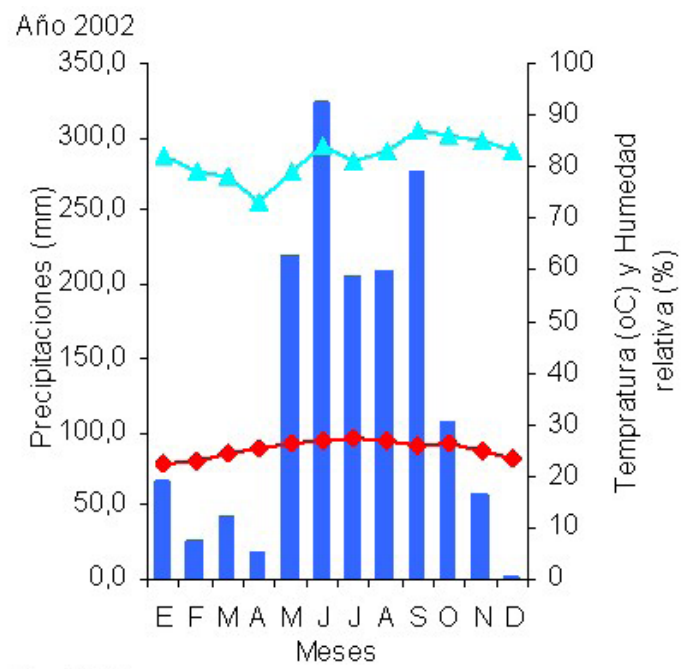

Año 2004
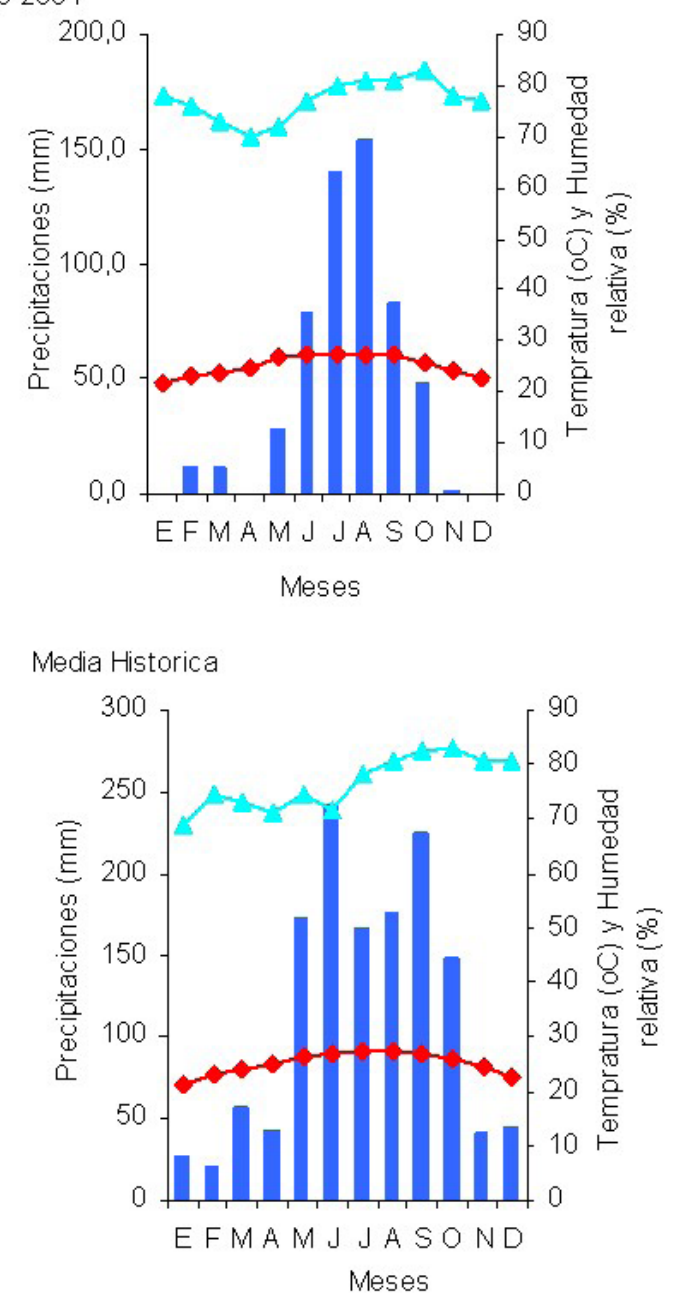

Precipitaciones $\rightarrow-$ Temperatura

$\rightarrow-\mathrm{Hr}$

Figura 1. Comportamiento de algunas variables climatológicas durante la etapa experimental (2001-2005). 
- Porosidad de aireación (Va): cálculo a partir de la porosidad total y la humedad de capacidad de campo: $\mathrm{Va}=P_{t}-d_{v} * \mathrm{Wcc}$

donde la Wcc (humedad a capacidad de campo) se determinó por el método de campo, inundando con agua una parcela de suelo representativa del área de estudio.

- Coeficiente de estructura $\left(\mathrm{K}_{\mathrm{e}}\right)$ : método de Sávinov según Kaúrichev (1984), de los resultados de este análisis se determinó el coeficiente de estructura (Ke): $K e=\frac{c}{g}$

Donde: c: contenido de agregados de diámetro comprendidos entre $10 \mathrm{y}$

$0.25 \mathrm{~mm}$; g: agregados de diámetro $>10 \mathrm{~mm}$ y $<0.25 \mathrm{~mm}$.

- Estabilidad estructural: tamizado en húmedo, resultando el \% de agregados estables al agua (Ae) $>0.25 \mathrm{~mm}$.

- Velocidad de infiltración del agua: método de los anillos concéntricos, con humedad inicial del suelo correspondiente al límite productivo.

Los valores detectados en las propiedades físicas del suelo, se les realizó un análisis factorial. La existencia o no de diferencias entre las medias de los tratamientos, se corroboró a través de la prueba de Tukey para $\mathrm{p}<0.05$. Todos los análisis se realizaron con ayuda del paquete estadístico STATGRAPHIC versión Plus 5.0, soportada sobre el sistema operativo Windows.

\section{Resultados y discusión}

Efecto de las variantes experimentales sobre algunas propiedades físicas del suelo

La densidad de la fase sólida (Tabla 1) no experimentó variación alguna para los diferentes tratamientos, durante el período evaluado, lo que permite afirmar que esta es una propiedad poco dinámica que depende, fundamentalmente, de la composición química y mineralógica del suelo. Trabajos investigativos realizados por Cabrera et. al. (2002) y Armengol (2006), aplicando diferentes enmiendas orgánicas por cuatro años consecutivos corroboraron que la materia orgánica no introdujo cambios en la densidad de la fase sólida, por lo que consideran esta propiedad como una de las más estables del suelo.

Sin embargo, la densidad del suelo experimentó diferencias significativas entre los tratamientos, con énfasis en los años posteriores al establecimiento de ambas coberturas. Los valores se alcanzaron más bajos en el tratamiento donde se estableció Teramnus labialis, lo que confirma el efecto positivo que ejerce esta alternativa al propiciar incrementos en los contenidos de materia orgánica (FONTES, 2007). Ello favoreció el volumen del espacio poroso total, y por consiguiente, produjo una disminución de la densidad del suelo.

Otra razón que justifica el empleo de alternativas que garanticen la protección del suelo es el hecho de que, según la tecnología actualmente utilizada en el manejo de plantaciones citrícolas, conlleva a que la compactación y degradación del suelo se intensifique por el uso excesivo de maquinarias para garantizar un suelo desnudo durante toda la plantación, todo lo cual trae consigo empeoramiento de las propiedades físicas del mismo, relacionadas con las disminuciones de los contenidos de materia orgánica y el papel que desempeña la misma según Ferrotti y Luciano (2005). Esta evidencia confirma la necesidad de introducir el uso de coberturas, práctica no seguida actualmente, en plantaciones comerciales de cítricos en Cuba. Investigaciones realizadas por Peña (2000) y Cabrera et. al. (2002), recomiendan el empleo de enmiendas o mejoradores para suelos con labranza intensiva. 
Tabla 1. Comportamiento de algunas propiedades físicas del suelo, al finalizar el primer y cuarto año de la fase experimental, en los primeros $0.20 \mathrm{~m}$.

\begin{tabular}{|c|c|c|c|c|c|c|c|c|c|c|}
\hline \multirow{3}{*}{ Tratamientos } & \multicolumn{4}{|c|}{ kg. $\mathrm{m}^{-3} \cdot \mathbf{1 0}^{3}$} & \multicolumn{6}{|c|}{$\mathrm{m}^{3} \cdot \mathrm{kg}^{-1} \cdot 10^{-5}$} \\
\hline & \multicolumn{2}{|c|}{$\left(\rho_{s}\right)$} & \multicolumn{2}{|c|}{$d_{v}$} & \multicolumn{2}{|c|}{ Wec } & \multicolumn{2}{|c|}{$\mathbf{P t}$} & \multicolumn{2}{|c|}{ Va } \\
\hline & Inicio & Final & Inicio & Final & Inicio & Final & Inicio & Final & Inicio & Final \\
\hline $\begin{array}{c}\text { Cobertura } T . \\
\text { labialis }\end{array}$ & 2.55 & 2.55 & $1.17 \mathrm{~b}$ & $0.96 \mathrm{~d}$ & $30.01 \mathrm{c}$ & $34.12 \mathrm{a}$ & $54.1 \mathrm{c}$ & $62.3 \mathrm{a}$ & $18.7 \mathrm{c}$ & $29.5 \mathrm{a}$ \\
\hline $\begin{array}{c}\text { Cobertura } \\
\text { natural }\end{array}$ & 2.56 & 2.55 & $1.20 \mathrm{~b}$ & $1.09 \mathrm{c}$ & $28.75 d$ & $31.4 \mathrm{~b}$ & $53.1 \mathrm{~cd}$ & $57.2 \mathrm{~b}$ & $18.6 \mathrm{c}$ & $22.9 \mathrm{~b}$ \\
\hline Suelo desnudo & 2.55 & 2.56 & $1.25 \mathrm{a}$ & $1.24 \mathrm{a}$ & $27.0 \mathrm{e}$ & $27.12 \mathrm{e}$ & $50.9 \mathrm{e}$ & $51.5 \mathrm{de}$ & $17.2 \mathrm{~d}$ & $17.8 \mathrm{~cd}$ \\
\hline Es $\bar{X}$ & \multicolumn{2}{|c|}{$0.002 \mathrm{~ns}$} & \multicolumn{2}{|c|}{0.02} & \multicolumn{2}{|c|}{0.06} & \multicolumn{2}{|c|}{0.64} & \multicolumn{2}{|c|}{0.45} \\
\hline
\end{tabular}

Cobertura T. labialis; Cobertura natural; Suelo desnudo; Inicio: al finalizar el primer año experimental (2002); Final: al finalizar el cuarto año (2005). $\left(\rho_{s}\right)$ : densidad de la fase sólida; $\mathrm{d}_{\mathrm{v}}$ : densidad de suelo; Wcc: humedad capacidad de campo; Pt: porosidad total, Va: volumen específico de aire. Medias con letras no comunes, para un mismo indicador y profundidad, difieren según Tukey ( $\mathrm{p}<$ $0,05)$. Es $\overline{\mathbf{X}}$ : error estándar de media.

A pesar de que para Rodríguez (2005) la porosidad total de los suelos Ferralíticos se caracteriza por ser alta, relacionada con el drenaje y movimiento del agua en el perfil, así como con el intercambio gaseoso y el crecimiento radical, lo que en la investigación se comprueba con el uso de coberturas, y más aún en la de T. labialis, la que mejora la distribución del espacio poroso total, la que se incrementó en un $8.2 \%$.

Un análisis de la humedad a capacidad de campo, el volumen específico total de poros y el volumen específico de aire, permite constatar que existieron diferencias significativas entre los tratamientos para cada uno de estos indicadores. Los valores más altos se encontraron con la cobertura de Teramnus labialis, tratamiento en el que se logró además un mejor estado estructural, comportamiento supeditado a los incrementos de materia orgánica y a las mejores condiciones de humificación que se producen en esta alternativa (FONTES, 2007).

Los resultados confirman las posibilidades que ofrece esta leguminosa, la que propicia una mayor retención de humedad a partir de la cobertura que desarrolla durante su establecimiento, pudiéndose inferir las disminuciones de la temperatura del suelo, el mejoramiento de las condiciones de vida para la macrobiota acompañante, entre otras, la que se hace superior si se compara con el tratamiento donde se encontraba el suelo desprovisto de vegetación.

Las mejores condiciones estructurales se presentaron en el tratamiento donde se estableció la cobertura de T. labialis (Tabla 2), las que difirieron significativamentedelasotrasalternativasempleadas. Ello se explica a partir de los incrementos que ejerció la cobertura sobre los contenidos de materia orgánica y otros nutrientes (RODRÍGUEZ, 2005). Por tal motivo se supone que este comportamiento se debe a que la materia orgánica, conjuntamente con el hierro, forma microagregados estables en la parte superior del perfil como parte de la formación natural del suelo y que éstos tienden a descomponerse por la influencia antropogénica, cuando el suelo es sometido al cultivo intensivo, afirmación realizada por Morell (2005).

Un efecto contrario manifestó el tratamiento de suelo desnudo, donde el manejo tradicional utilizado en las plantaciones citrícolas que se desarrollan sobre suelos Ferralíticos, es la causa fundamental que propicia su degradación física, aspecto referido por Cabrera (2000) y Armengol (2006), quienes demostraron que la adición de determinadas dosis de residuos orgánicos, en diferentes tipos 
de suelo, provocó incrementos del coeficiente de estructura, lo cual repercutió positivamente en su acondicionamiento hidrofísico.

El efecto de los tratamientos evaluados sobre la estabilidad de los agregados estables al agua $>0.25$ $\mathrm{mm}$ al primer y cuarto año de la fase experimental, en los primeros $0.20 \mathrm{~m}$, se aprecian en la Tabla 3.

Los resultados obtenidos muestran que el efecto acumulativo de la biomasa depositada por la cobertura de leguminosa al suelo, favorece las características estructurales del mismo e incidió en el aumento de los agregados agronómicamente más valiosos y en la estabilidad de los agregados al agua, aspectos todos que influyeron favorablemente en el crecimiento y desarrollo, manifestado en el rendimiento del naranjal, atribuido a lo informado por Carvalho, Leone y Oliverira (2007), quienes señalan que la materia orgánica influye en la estabilidad hídrica de los agregados del suelo, razón por la cual son inversos los resultados alcanzados donde se encontraba el suelo sin proteger.

Resultados similares informa Morell (2005), quién encontró, en un suelo bajo bosque, hay un predominio de agregados de 7-2 $\mathrm{mm}(57.8 \%)$ con una alta porosidad de los mismos $(42.6 \%)$ y una elevada estabilidad estructural (91.9\%); donde la fracción de 5-2 mm, es la más resistente a la acción destructora del agua.

Tabla 2. Composición de los macroagregados, $(\mathrm{Ke})$ en el suelo, al finalizar el primer y cuarto año de la fase experimental, en los primeros $0.20 \mathrm{~m}$.

\begin{tabular}{|c|c|c|c|c|c|c|c|c|c|c|c|c|c|c|}
\hline \multirow{3}{*}{ Trat. } & \multicolumn{12}{|c|}{ Composición de macroagregados por fracciones (\%). } & \multirow{2}{*}{\multicolumn{2}{|c|}{ Ke }} \\
\hline & \multicolumn{2}{|c|}{$>10 \mathrm{~mm}$} & \multicolumn{2}{|c|}{$10-7 \mathrm{~mm}$} & \multicolumn{2}{|c|}{ 7-5 mm } & \multicolumn{2}{|c|}{ 5-3 mm } & \multicolumn{2}{|c|}{ 3-0.25 mm } & \multicolumn{2}{|c|}{$<0.25 \mathrm{~mm}$} & & \\
\hline & Inicio & Final & Inicio & Final & Inicio & Final & Inicio & Final & Inicio & Final & Inicio & Final & Inicio & Final \\
\hline $\mathrm{T}_{1}$ & 11.3 & 9.0 & 18.2 & 21.82 & 20.2 & 24.64 & 28.30 & 30.4 & 17.0 & 9.36 & 5.0 & 4.78 & $5.13 \mathrm{c}$ & $6.25^{\mathrm{a}}$ \\
\hline $\mathrm{T}_{2}$ & 13.12 & 9.9 & 18.0 & 19.6 & 20 & 24.6 & 30.0 & 35.04 & 13.6 & 5.8 & 5.28 & 5.06 & $4.43 \mathrm{~d}$ & $5.68 \mathrm{~b}$ \\
\hline $\mathrm{T}_{3}$ & 16.2 & 13.6 & 21.28 & 14.28 & 18.74 & 19.8 & 22.46 & 31.8 & 17.66 & 14.22 & 3.66 & 6.27 & $4.03 \mathrm{e}$ & $4.03 \mathrm{e}$ \\
\hline Es $\bar{X}$ & & & & & & & - & & & & & & & 20 \\
\hline
\end{tabular}

Tratamientos: $\mathrm{T}_{1}$. Cobertura de Teramnus labialis, $\mathrm{T}_{2}$. Cobertura natural, $\mathrm{T}_{3}$. Suelo desnudo. Inicio: al finalizar el primer año experimental(2002); Final: al finalizar el cuarto año (2005). Medias con letras no comunes, para un mismo indicador, difieren según Tukey $(\mathrm{P}<0,05)$. Es $\overline{\mathbf{X}}$ : error estándar de la media.

Tabla 3. Porcentaje de agregados estables al agua (Ae) $>0.25 \mathrm{~mm}$ al primer y cuarto año de la fase experimental, en los primeros $0.20 \mathrm{~m}$.

\begin{tabular}{|c|c|c|c|c|c|c|c|c|c|c|c|c|}
\hline \multirow{3}{*}{ Trat. } & \multicolumn{10}{|c|}{ Porcentajes de agregados estables al agua por fracciones } & \multirow{2}{*}{\multicolumn{2}{|c|}{ Ae $(\%)$}} \\
\hline & \multicolumn{2}{|c|}{ 10-7 mm } & \multicolumn{2}{|c|}{ 7-5 mm } & \multicolumn{2}{|c|}{$5-3 \mathrm{~mm}$} & \multicolumn{2}{|c|}{ 3-0.25 mm } & \multicolumn{2}{|c|}{$<0.25 \mathrm{~mm}$} & & \\
\hline & Inicio & Final & Inicio & Final & Inicio & Final & Inicio & Final & Inicio & Final & Inicio & Final \\
\hline $\mathrm{T}_{1}$ & np & np & $\mathrm{np}$ & $\mathrm{np}$ & 48.05 & 56.8 & 30.53 & 33.34 & 21.42 & 9.86 & $78.58 \mathrm{c}$ & $90.14 \mathrm{a}$ \\
\hline $\mathrm{T}_{2}$ & np & np & np & $\mathrm{np}$ & 29.85 & 32.95 & 46.53 & 52.19 & 23.62 & 14.86 & $77.71 \mathrm{c}$ & $85.14 b$ \\
\hline $\mathrm{T}_{3}$ & $\mathrm{np}$ & $\mathrm{np}$ & $\mathrm{np}$ & $\mathrm{np}$ & 31.2 & 29.6 & 41.63 & 43 & 27.4 & 27.17 & $72.6 \mathrm{~d}$ & $72.8 \mathrm{~d}$ \\
\hline Es $\bar{X}$ & & & & & & & & & & & 1.5 & \\
\hline
\end{tabular}

Tratamientos: $\mathrm{T}_{1}$. Cobertura de Teramnus labialis, $\mathrm{T}_{2}$. Cobertura natural, $\mathrm{T}_{3}$. Suelo desnudo. Inicio: al finalizar el primer año experimental (2002); Final: al finalizar el cuarto año (2005); np: no presente. Medias con letras no comunes, para un mismo indicador, difieren según Tukey $(\mathrm{P}<0,05)$. Es $\overline{\mathbf{X}}$ : error estándar de la media. 
Los cambios que en el estado estructural del suelo tuvieron lugar durante el establecimiento de las coberturas incidieron en las magnitudes físicas relacionadas con el movimiento de agua en el suelo, específicamente en la velocidad de infiltración. Las curvas de la velocidad de infiltración mostradas en la figura 2 indican correspondencia entre el mejor estado estructural y el incremento del volumen específico de poros, lo que favorece aumentos significativos de la velocidad de infiltración del agua en el suelo en las variantes de cobertura con respecto al suelo desnudo, diferencia que se relaciona con el incremento de la materia orgánica que en dichas condiciones se produjo.

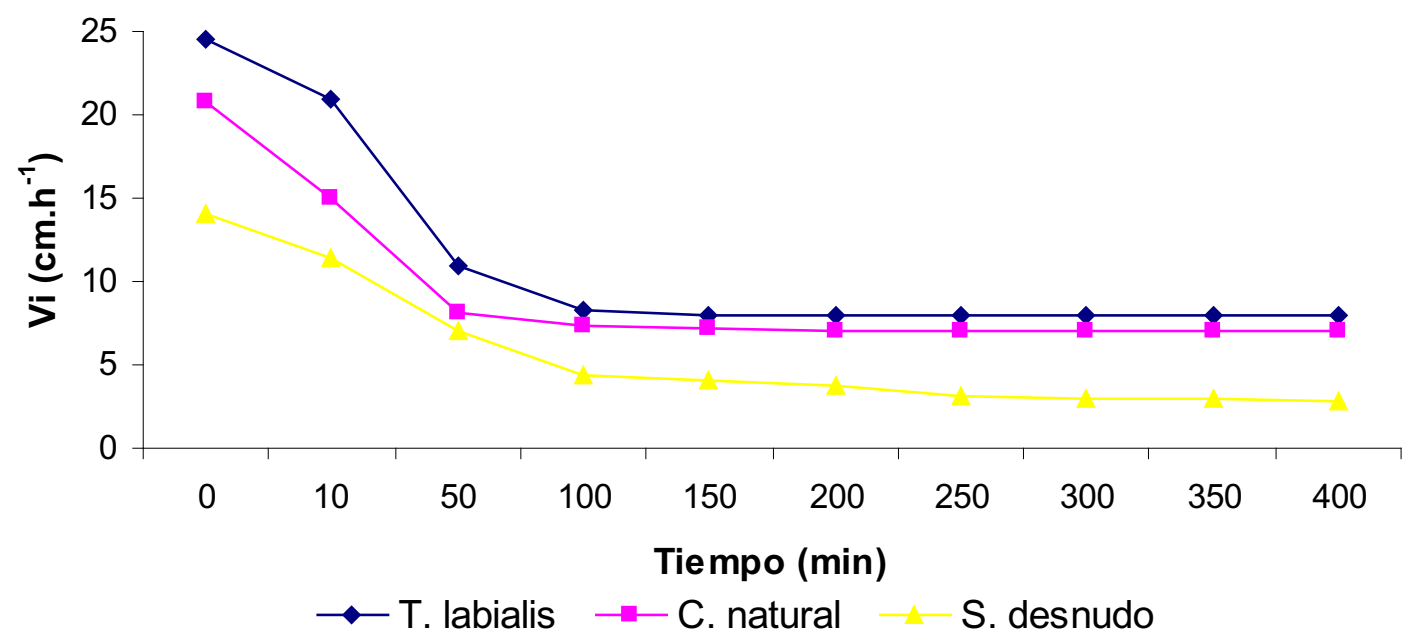

Figura 2. Velocidad de infiltración del agua (Vi) en las diferentes tratamientos estudiados al finalizar el cuarto año de la etapa experimental.

Las variaciones de la velocidad de infiltración, conjuntamente con los cambios ocurridos en la cuantía del agua conservada en el primer horizonte, en los tratamientos con cobertura, muestran las condiciones bajo las cuales se retuvo y se produjo el movimiento del agua en el suelo, lo que repercutió positivamente en el suministro de agua a la planta, resultados que corroboran con lo obtenidos por Pérez-Carmenate (1998), Gutiérrez (2001) y Rodríguez (2005), quienes coinciden en afirmar que el empleo de leguminosas, como cobertura vegetal, resulta un factor significativo en la conservación del agua en los suelos, en la mejora de la infiltración, el drenaje y en la disminución de la evaporación, elementos todos favorables al cultivo base.

\section{Conclusión}

Los incrementos en los contenidos de humedad, el volumen específico total de poros y de aire, el coeficiente de estructura, así como el porcentaje de agregados estables al agua, en el suelo, donde se establece la cobertura de Teramnus labialis, demuestran la eficiencia de la cobertura vegetal en estas plantaciones citrícolas.

\section{Referencias}

ARMENGOL, J. E. Modificaciones inducidas por la vinaza en las propiedades fisicas y químicas de vertisoles de caña de azúcar en la zona norte de Ciego de Ávila. 2006. Tesis (Doctor en Ciencias Agrícolas) - Universidad de Ciego Ávila: UNICA, 2006. 
BORROTO, A.; PÉREZ, R.; MAZORRA, C.; FONTES, D.; BORROTO, M.; CUBILLAS, N.; HERNÁNDEZ, N.; LÓPEZ, J. L.; DE LA ROSA, A.; TAPIA, L.; RODRÍGUEZ, L.; GUTIERREZ, I.; MARTÍNEZ, G.; RODRÍGUEZ, I. Tecnologías para la obtención de carne en sistemas de agricultura sostenible. Ciego de Ávila, 2000. Programa Nacional de Investigación en la Producción de Alimento Animal por Vía Biotecnológica y Sostenible. Informe final CITMA, Cuba.

CABRERA, S. Nuevo enfoque agrofísico para la evaluación del mejoramiento de los suelos (en el ejemplo de los vertisoles). 2000. Tesis (Doctor en Ciencias Agrícolas) - Universidad de Ciego Ávila: UNICA, 2000.

CABRERA, S.; PÉREZ, C.; PLA, E.; DOMÍNGUEZ, J.; ABRÉU, O. Influencia de la materia orgánica sobre los índices estructurales fundamentales de un vertisol y los rendimientos de la caña de azúcar. Revista Ciencias Técnicas Agropecuarias, Habana, v. 8, n. 3, p. 55-58. 2002.

CARVAlHO, J. E.; LEONE, C.; OLIVEIRA, J. Manejo del suelo y cobertura vegetal en frutales. En: MEMORIAS DEL SIMPOSIO INTERNACIONAL DE FRUTICULTURA TROPICAL Y SUBTROPICAL, 2. 2007, Habana. Anais...Habana, 2007.

CLAVEL, N. Contribución de la cobertura con leguminosas forrajeras a la conversión a orgánico de un agroecosistema citrícola. 2004. Tesis (Master en Pastos y Forrajes) - Universidad de Matanzas "Camilo Cienfuegos” EEPF “Indio Hatuey”, 2004.

DRIESSEN, P., DECKERS, J.; NACHTERGAELE, F. O. Lecture Notes on the Major Soils of the World. Rome: FAO, 2001. 334 p.

FERROTTI, F.; LUCIANO, C. Soil organic matter and soil nitrogen fraction as affected by crop rotation in rainfed mediterranean conditions. En: INTERNATIONAL CONFERENCE OF GLOBAL SOIL CHANGE, 2005, Ciudad de México. Anais... Ciudad de México, 2005.

FONTES, D. Beneficios agroproductivos de Teramnus labialis (L.f.) Spreng. como cobertura en plantaciones citrícolas. 2007. Tesis (Doctor en Ciencias Agrícolas) Universidad de Ciego Ávila: UNICA, 2007.

GUTIÉRREZ, I. Efectos de coberturas vivas de leguminosas en una plantación de naranja Valencia late. 2001. Tesis (Master en Citricultura tropical) Universidad de Ciego Ávila: UNICA, 2001.
GUTIÉRREZ, I.; FELIPE, M.; PÉREZ, R.; FONTES, D.; VERNON, D. Efectos de una cobertura viva de Teramnus labialis (L.F.) Sprengel sobre las arvenses en campos citrícolas. Fitosanidad, Habana, v. 10, n. 1, p. 49-53, 2006.

HERNÁNDEZ, A.; PÉREZ, J. M.; BOSH, I. D.; RIVERO, L.; DURAN J. L.; CID, G. PONCE, L. D. Nueva Versión de la Clasificación Genética de los Suelos de Cuba. Habana: Ministerio de la Agricultura, 1999. (Formato digital).

KAÚRICHEV, I. S. Prácticas de Edafología. Moscú: MIR, 1984. 286 p.

LORENZO, R. Efecto del residuo de la producción de la levadura torula sobre el estado físico de los suelos Ferralíticos amarillentos dedicados a la caña de azúcar. 1992. Tesis (Doctor en Ciencias Agrícolas) - Universidad Ciego de Ávila: ISACA, 1992. 123 p.

MORELL, F. Degradación de las propiedades agrobiológicas de los suelos Ferralíticos Rojos lixiviados por la influencia antrópica y su respuesta agroproductiva al mejoramiento. 2005. Tesis (Master en Nutrición de las Plantas y Biofertilizantes) - Instituto Nacional de Ciências Agrícolas, 2005.

NAVIA, Y. Uso de la leguminosa herbácea Teramnus labialis como cobertura en el cultivo de la guayaba. 2005. Tesis (Master en Ciencias Agrícolas) - Universidad Ciego de Ávila: UNICA, 2005.

PEÑA, C. M. Mejoramiento de la tecnología de diseño y explotación de sistemas de microriego en cítricos. 2000. Tesis (Doctorado en Ciencias Agrícolas) - Universidad Ciego de Ávila: UNICA, 2000.

PÉREZ CARMENATE, R. Leguminosas herbáceas perennes una alternativa para la diversificación de las fincas citrícolas. 1998. Tesis (Master en Pastos y Forrajes) - Universidad de Matanzas "Camilo Cienfuegos"EEPF "Indio Hatuey", 1998.

PÉREZ, A.; ROLO, R. Efecto de las dosis de fósforo y potasio sobre la producción de semillas de leguminosas. II. Lablab purpureus, Rongai, v. 21, p. 219, 1998.

RODRÍGUEZ, O. Efectos de una cobertura de Teramnus labialis en una plantación de naranja Valencia late. 2005. Tesis (Graduação Ingeniero Agrónomo) - Universidad Ciego de Ávila: UNICA, 2005.

SOIL SURVEY STAFF. Claves para la Taxonomia de Suelos. Chapingo: Colegio de Postgraduados, 2003. 
